\title{
One-Year, Efficacy and Safety Open Label Study, with a Single Injection of a New Hyaluronan for Knee OA: The SOYA Trial
}

\author{
Carlos Gavín (D) \\ Francisco J Blanco (D) ${ }^{2}$ \\ José L Pablos ${ }^{3}$ \\ Miguel A Caracuel ${ }^{4}$ \\ José Rosas ${ }^{5}$ \\ Enrique Gómez-Barrena ${ }^{6}$ \\ Francisco Navarro ${ }^{7}$ \\ María Pilar Coronel $^{8}$ \\ Mercedes Gimeno ${ }^{8}$ \\ 'Hospital Universitario Fundación \\ Alcorcón, Madrid, Spain; ${ }^{2}$ Complejo \\ Hospitalario Universitario aCoruña, La \\ Coruña, Spain; ${ }^{3}$ Hospital Universitario 12 \\ de Octubre, Madrid, Spain; ${ }^{4}$ Hospital \\ Universitario Reina Sofía, Córdoba, Spain; \\ ${ }^{5}$ Hospital Marina Baixa, Villajoyosa, \\ Alicante, Spain; ${ }^{6}$ Hospital Universitario La \\ Paz, Madrid, Spain; ${ }^{7}$ Hospital General \\ Universitario de Elche, Alicante, Spain; \\ ${ }^{8}$ Meiji Pharma Spain, Alcalá de Henares, \\ Madrid, Spain
}

Correspondence: Mercedes Gimeno Clinical Development Department, Meiji Pharma Spain, Av Madrid 28802, Alcalá de Henares, Madrid, Spain

Tel +34918 870980

Email m.gimeno@meiji.es
Purpose: To assess the efficacy and safety of a single injection of a new formulation of hyaluronic acid (MPS-HA2\%) in patients with symptomatic knee osteoarthritis after 12 months' follow-up.

Patients and Methods: Prospective, single-arm, multicentre, open-label, 12-month follow-up study. Patients with Kellgren-Lawrence (KL) 2-3 and visual analogue scale (VAS) pain scores of $\geq 40-<80 \mathrm{~mm}$ received a single injection of MPS-HA2 $\%$. The primary outcome was the reduction in VAS pain scores from baseline, and the secondary outcomes were the Western Ontario and McMaster (WOMAC) Universities Osteoarthritis Index, the minimum clinically important improvement (MCII), and patient and investigator global assessments (PGA, IGA) measured on 5-point Likert scale. Adverse events were recorded throughout the study for safety purposes.

Results: A total of 101 patients (mean age: 68 years; 74\% female; and 78\% overweight) were included. The mean reduction in pain at 12 months was $37.7 \%$; the total WOMAC score improved by $36.5 \%$ and the pain, stiffness and physical function subscores returned improvements of $32.1 \%, 34.1 \%$ and $32.7 \%$, respectively ( $\mathrm{p}=0.0001$ with respect to baseline). At 12 months, a statistically significant $62.2 \%$ of patients obtained an improvement equal to or greater than the MCII. The mean PGA score at baseline was 2.44 and 1.46 at 12 months $(\mathrm{p}<0.05)$, and the mean IGA scores at equivalent timepoints were 2.29 and $1.48(\mathrm{p}<0.05)$. Fourteen patients received a second injection at the 6-month follow-up visit. Eight patients reported a total of 12 treatment-related adverse events that were local, non-serious and of mild-to-moderate intensity.

Conclusion: With just a single intra-articular injection, this not controlled trial suggests that MPS-HA2\% is effective 12 months after the procedure in most cases. Patient tolerability and safety were both optimal (NCT03852914).

Keywords: osteoarthritis, pain, hyaluronic acid, viscosupplementation

\section{Introduction}

Osteoarthritis (OA) of the knee is a common joint disorder in which progressive cartilage destruction, osteophyte formation, subchondral sclerosis and synovitis lead to articular degeneration. ${ }^{1-3}$ It is a multifactorial disease with genetic alterations, gender and ageing playing crucial roles in the processes associated with cartilage and joint degeneration and therefore in the development of OA. ${ }^{4,5}$ The main manifestations include pain, physical dysfunction and markedly reduced quality of life. ${ }^{6}$ It constitutes a high socioeconomic burden, with joint replacement responsible for most of the direct healthcare costs, besides the indirect costs arising from productivity losses, premature death and early retirement. ${ }^{7}$ Due to the 
multifactorial nature, the different treatment targets and high frequency of associated comorbidities, researchers are yet to identify the ideal therapy for OA. This has led to a long-term multimodal approach including different pharmacological and nonpharmacological therapeutic interventions depending on the stage of the disease and each patient's characteristics. ${ }^{4,5,7-9}$

Hyaluronic acid (HA) is a naturally occurring polysaccharide found within synovial fluid and produced by typeB synoviocytes, fibroblasts and chondrocytes; it is widely distributed in connective tissues and a primary component of articular fluid. It provides nutrients for articular cartilage, lubricates articulation and absorbs strains. The molecular weight (MW) and rheological properties of the HA in the synovial cavity change as we age and in the case of joint disorders. $^{10}$

Viscosupplementation of the synovial fluid with intra-articular HA injections was first included in the therapeutic arsenal at the end of the 20th century, with the intention being to restore the concentration and molecular weight distribution of HA in the synovial fluid. However, there is now evidence that the clinical benefit of HA in OA is not only due to its mechanical properties; several studies have found that it also exerts a chondroprotective effect, promotes proteoglycan and glycosaminoglycan synthesis, and has anti-inflammatory, mechanical, subchondral and analgesic properties. ${ }^{11}$ Despite some discrepancies between scientific societies regarding the widespread use of intra-articular $\mathrm{HA}$ to treat knee OA, several systematic reviews and metaanalyses published recently concluded that viscosupplementation is a viable treatment option that provides a clinically important reduction in pain and improvement in function. ${ }^{12-19}$ This is especially true when the treatment is adequately tailored according to patient characteristics, ${ }^{20,21}$ with effects lasting more than those of intra-articular corticosteroids ${ }^{22-26}$ and with a better safety profile than other treatments for OA. ${ }^{19,24,27-30}$

Treatment with HA has traditionally consisted of several intra-articular injections administered at weekly intervals. However, there is an ever-increasing demand for shorter treatments, resulting in less visits and costs for both patients and healthcare providers. Furthermore, the use of single-dose treatments would reduce the potential risk of local adverse events related to the administration procedure. Different approaches have been developed in order to obtain effects that are as durable as those of multiple-injection treatments, such as greater injection volumes, higher concentrations, changes in the molecular structure (crosslinking) or the inclusion of different additives, but there is still no clear consensus about the impact of these modifications on the clinical results. ${ }^{31}$

Most studies of knee OA involving single-injection products were designed to follow the patients for no more than 6 months; ${ }^{32}$ therefore, in most cases, the scarcity of results makes it hard to comment on the long-term duration of the effects.

The SOYA (Symptomatic Osteoarthritis one Year Assessment) study was designed to investigate the efficacy and safety of a new formulation of HA in the treatment of patients with symptomatic knee OA, focusing particularly on the effects 12 months after a single injection.

\section{Patients and Methods}

\section{Ethics and Registration}

The trial was conducted in accordance with Good Clinical Practices and in compliance with the principles of the Declaration of Helsinki. Approval was obtained from the Alcorcón Hospital Institutional Review Board (Alcorcón, Madrid, Spain) (approved on 6th of September 2018, minutes $n^{\circ}$ 10/18) and the Spanish Agency of Medicines and Medical Devices. All patients provided their written informed consent to participate in this trial. The study was registered at ClinicalTrials.gov with the identifier NCT03852914.

\section{Design}

The study followed a prospective, single-arm, open-label design with a 12-month follow-up. Patients deemed eligible at screening completed a 14-day washout period prior to administration. Each patient then received a single intraarticular injection of MPS-HA2\%, a new product consisting of a $2 \% \mathrm{wt}$./vol solution of medium MW $(1000 \mathrm{kDa})^{33}$ HA obtained by fermentation and without any chemical modification, dosed in pre-filled syringes containing $98 \mathrm{mg}$ of HA. Patients were followed for 12 months, with a telephone contact at 3 months for safety purposes and follow-up visits at 6 and 12 months (Figure 1). At 6 months, patients were offered a second injection if and when they met all the criteria established for re-treatment : 1) a reduction in pain from baseline on VAS $<$ MCII; 2) the patient's overall opinion; and 3) no treatment-related adverse events after the first administration; and were followed until completion of the initial 12 month period. Only paracetamol (up to $3 \mathrm{~g} /$ day) was permitted as rescue 

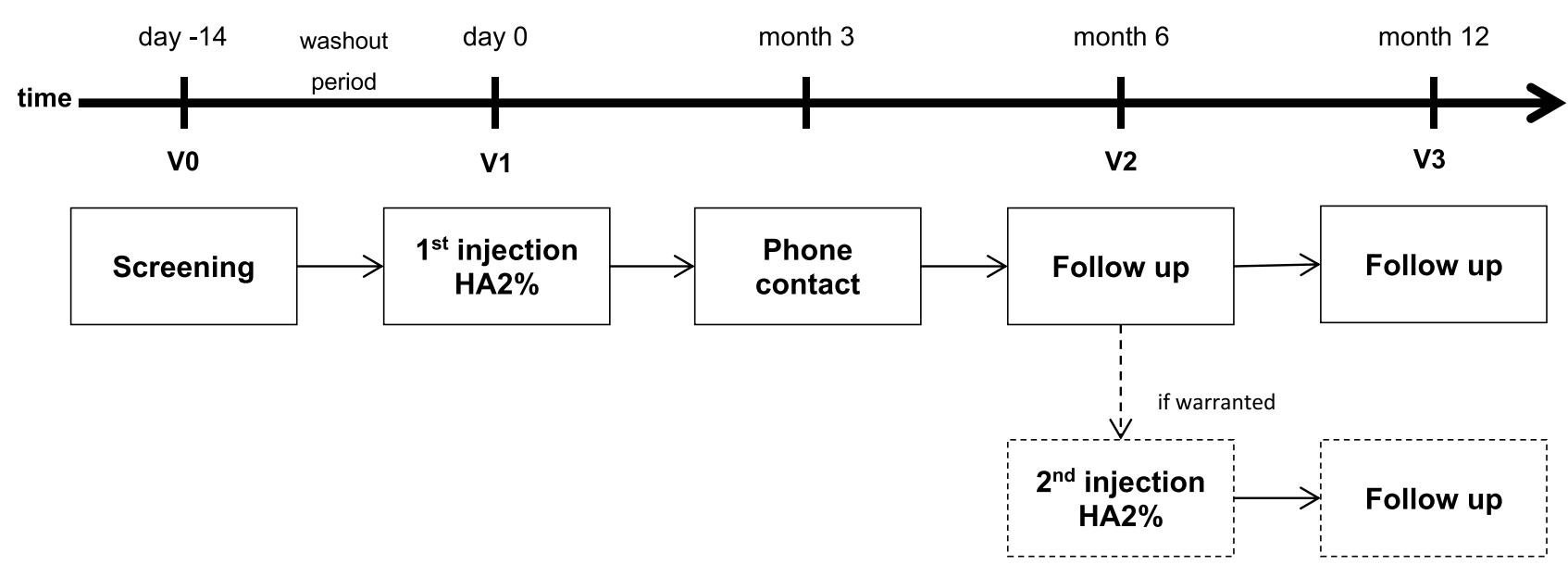

Figure I Study schedule.

medication for OA pain during the study and it had to be discontinued at least $24 \mathrm{~h}$ before visits.

\section{Study Population}

Eligible patients were men and women aged $\geq 45$ years who met the following inclusion criteria: diagnosis of primary $\mathrm{OA}$ of the knee in the medial/lateral tibiofemoral compartment according to ACR criteria; ${ }^{34}$ radiological grade 2-3 according to the Kellgren and Lawrence (KL) scale $^{35}$ confirmed by an anteroposterior X-ray; and VAS pain scores $\geq 40-<80 \mathrm{~mm}$ in the target knee and $\leq$ $20 \mathrm{~mm}$ in the contralateral knee. Patients had to be able to understand and comply with the study procedures and give their written consent to participate before entering in the study.

Exclusion criteria comprised a body mass index (BMI) $\geq 30 \mathrm{~kg} / \mathrm{m}^{2}$, a severe varus/valgus deformity $\left(>15^{\circ}\right)$ or patellofemoral pain/syndrome, previous surgery in the target knee including arthroscopy within the past year, severe inflammation of the target knee, an inflammatory joint disease, significant $\mathrm{OA}$ in other joints requiring pharmacological treatment, venous or lymphatic stasis in the relevant limb. Patients with the following treatments were also excluded: intra-articular HA (within the last year), intra-articular corticosteroids or joint lavage (last 3 months) and symptomatic slow-acting drugs for OA (SYSADOA). Finally, patients could not participate if they had known hypersensitivity to HA or paracetamol, were pregnant or breastfeeding, or if they were on a waiting list for surgery or awaiting a disability assessment.

\section{Outcome Measures}

We used patient-reported outcomes and derived measures to evaluate the efficacy of the treatment. The primary endpoint was a reduction in pain intensity measured on a $0-100 \mathrm{~mm}$ VAS, where 0 was the absence of pain and 100 was extreme pain. Secondary variables included the Western Ontario and McMaster (WOMAC) Universities Osteoarthritis Index total and subscale scores for pain (WOMAC A), stiffness (WOMAC B) and physical function (WOMAC C); all were measured on a VAS and normalized. Similarly, the percentage of patients who achieved the minimum clinically important improvement (MCII), ${ }^{36}$ defined as a relative improvement over baseline of $\geq 20 \%$ in VAS scores, was another secondary endpoint. The patient global assessment (PGA) and investigator global assessment (IGA) were measured on a 5-point Likert scale where 0 was the best condition and 4 the worst. Adverse events (AEs) were recorded from screening until the end of the study.

\section{Statistics}

A sample size of at least 93 patients was necessary to provide sufficient evidence that MPS-HA2\% is effective in the treatment of knee OA with a $90 \%$ power and considering a dropout rate of $35 \%$ after 12 months' follow-up.

The statistical and dataset analyses were defined in the Statistical Analysis Plan. We considered the results in terms of three populations. The modified intention-totreat (mITT) population included all patients who received at least one dose of study treatment and completed at least one post-baseline efficacy assessment. The per-protocol 
(PP) population included all patients who completed the study without any major protocol deviations. Finally, the safety population comprised all patients who received at least one dose of the study treatment. The main study population for the analysis was the mITT, although the PP population was also analysed.

Quantitative variables were reported as mean, median, standard deviation, interquartile range, minimum, maximum and the total number of patients with available data. Qualitative variables were described through their distribution of absolute and relative frequencies. Student's $t$-test or Wilcoxon nonparametric tests were used depending on the type and distribution of each dataset.

The statistical analysis was performed with SAS ${ }^{\circledR}$ v 9.3 and significance was set at $\mathrm{p}<0.05$.

\section{Results}

\section{Patient Disposition}

Seven Spanish hospitals participated in the study between January 2019 and May 2020 and preselected 104 patients of whom three were found to be ineligible. Finally, 101 patients were included and a total of $83(82.2 \%)$ completed the 12-month follow-up. The mITT population comprised 98 patients that received the study treatment and completed at least one post-baseline assessment. At 12 months, the PP population consisted of 74 patients who had completed the 12-month follow-up without any major protocol deviations. Of the 101 eligible patients, 17 did not complete the study: 2 cases were due to AEs (neither were study related), 6 patients decided to dropout, and 9 were withdrawn because of protocol violations. One additional patient was lost to follow-up. The CONSORT flow diagram of the trial is shown in Figure 2.

\section{Demographic and Baseline}

\section{Characteristics}

The patients had a mean age of 68 years and $50 \%$ were between 63 and 74 years old. The female/male ratio was approximately $3 / 1$ and $98 \%$ of the patients were Caucasian. With respect to occupations, $51.49 \%$ of the patients were retired, $25.74 \%$ were homemakers and $19.80 \%$ were employed in different professions (information on occupation was unavailable for 3 patients). The sample had a mean weight of $71.56 \mathrm{~kg}$ and a height of $1.62 \mathrm{~m}$. The mean BMI was $27.05 \mathrm{~kg} / \mathrm{m}^{2}$ and $78.22 \%$ of the patients were overweight. Nearly two-thirds $(65.35 \%)$ of the sample had three or more comorbidities

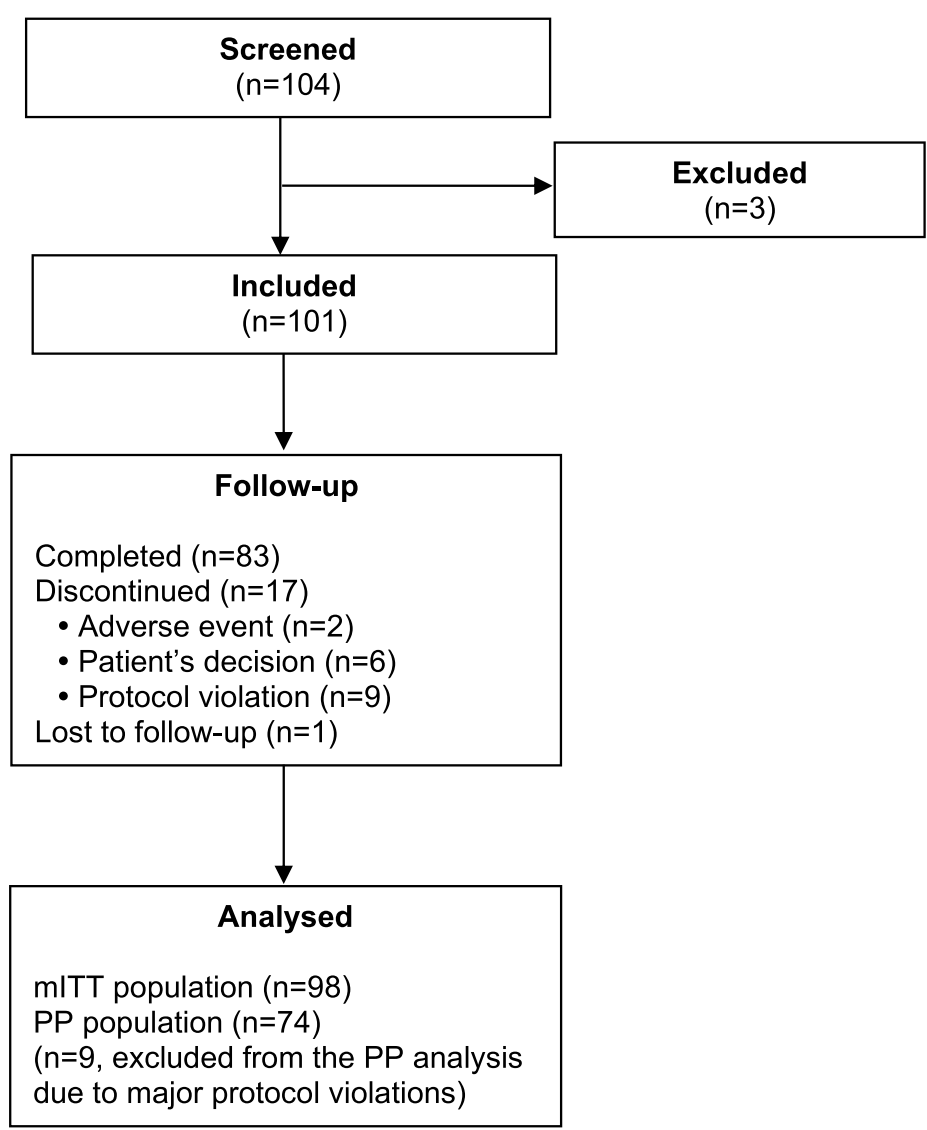

Figure 2 Flow diagram of the study.

and only $4 \%$ reported having no concomitant diseases. The most common comorbidities at inclusion were either related to the cardiovascular system or metabolic diseases, with frequencies of $60.40 \%$ and $53.47 \%$, respectively.

The mean time elapsed since the diagnosis of OA was 4.1 years (range: 1-28 years). Regarding the KL radiological grade, $57.4 \%$ of the subjects were grade 3 . Knee OA was unilateral in $26.70 \%$ of cases. All patients had predominantly tibiofemoral OA, while $62.38 \%$ also had some degree of patellofemoral compromise. Almost one-fifth (18.81\%) of patients had previously undergone surgery on the target knee (arthroscopy in 12 patients and meniscectomy in 7 others). The mean time since this surgery was 14.1 years.

Symptoms at baseline were moderate-to-severe, as determined through VAS pain scores, total WOMAC and WOMAC subscales A, B and C, as shown in Table 1.

Patients received one intra-articular injection of MPSHA2\% via a superolateral $(45.54 \%)$, lateral $(41.58 \%)$ or medial (11.88\%) approach. 
Table I Demographic and Baseline Characteristics of Eligible Patients $(n=101)$

\begin{tabular}{|c|c|c|}
\hline & Mean (SD) & n (\%) \\
\hline Women & & 75 (74.26\%) \\
\hline Age (years) & $68.04(9.05)$ & \\
\hline BMI $\left(\mathrm{kg} / \mathrm{m}^{2}\right)$ & $27.05(2.68)$ & \\
\hline Underweight & & I (0.99\%) \\
\hline Normal weight & & $20(19.80 \%)$ \\
\hline Overweight & & 79 (78.22\%) \\
\hline Obese & & I (0.99\%)* \\
\hline \multicolumn{3}{|l|}{ Comorbidities } \\
\hline Cardiovascular system & & $6 \mathrm{I}(60.40 \%)$ \\
\hline Metabolic and nutrition disorders & & $54(53.47 \%)$ \\
\hline Musculoskeletal and connective & & $34(33.66 \%)$ \\
\hline tissue disorders & & \\
\hline Nervous system & & $32(31.68 \%)$ \\
\hline Time since OA diagnosis (years) & $4.14(5.09)$ & \\
\hline Target knee (right) n (\%) & & $58(57.43 \%)$ \\
\hline \multicolumn{3}{|l|}{ Kellgren-Lawrence grade of target knee } \\
\hline Grade 2 & & $43(42.57 \%)$ \\
\hline Grade 3 & & $58(57.43 \%)$ \\
\hline VAS pain $(\mathrm{mm})$ & $63.86(11.60)$ & \\
\hline \multicolumn{3}{|l|}{ WOMAC (mm) } \\
\hline Total & $50.53(16.70)$ & \\
\hline Pain (A) & $49.81(17.42)$ & \\
\hline Stiffness (B) & $49.52(21.60)$ & \\
\hline Function (C) & $52.25(17.75)$ & \\
\hline
\end{tabular}

Note: *This patient was excluded after confirming their BMI.

Abbreviations: SD, standard deviation; BMI, body mass index; VAS, visual analogue scale; WOMAC, Western Ontario and McMaster Universities Osteoarthritis Index.

\section{Efficacy Results}

For the mITT population, the mean overall pain score (primary outcome) was $63.57 \mathrm{~mm}$ at baseline. By 6 months pain scores had reduced to a mean of $37.59 \mathrm{~mm}$, and this improvement was sustained over time with a mean score of $38.37 \mathrm{~mm}$ at 12 months. Very similar values were obtained among the PP population, with a mean pain scores of $63.82 \mathrm{~mm}$ at baseline and $36.97 \mathrm{~mm}$ and $36.61 \mathrm{~mm}$ at 6 and 12 months, respectively. These reductions were statistically significant $(\mathrm{p}<0.0001)$ and well above the $20 \%$ established as clinically relevant (Table 2 ).
Significant improvements were also observed in the total WOMAC score and the pain, stiffness and function subscales for both the mITT and PP populations; again, the improvement reported at 6 months was maintained at 12 months (Table 2).

The percentage of mITT patients who achieved MCII based on their VAS pain scores was $66.33 \%$ and $62.24 \%$ at 6 and 12 months, respectively. The equivalent figures in the PP population were $68.54 \%$ and $66.22 \%$.

The mean PGA score in the mITT population was 2.44 at baseline, 1.35 at 6 months and 1.46 at 12 months $(p<0.05)$. The scores were very similar for the PP population and the PGA improved significantly $(\mathrm{p}<0.05)$ at the end of the study. As for the IGA among the mITT population, the mean score was 2.29 at baseline, 1.06 at 6 months and 1.48 at 12 months $(\mathrm{p}<0.05)$. Staying with the mITT sample, the PGA was "better" or "much better" for $66.33 \%$ of patients after 6 months and $56.12 \%$ at 12 months. In the PP population the figures were very similar, with a result of better/much better reported by $67.42 \%$ of patients at 6 months and $62.16 \%$ at 12 months. Investigators rated the patients' condition as better/much better for $71.42 \%$ of cases at 6 months and $56.13 \%$ after 12 months' follow-up. The figures were very similar for the PP population where the IGA was better/much better for $74.16 \%$ of patients at 6 months and $63.51 \%$ at 12 months.

The additional analyses revealed that normal weight patients responded better to the treatment than overweight patients, although the differences did not reach statistical significance $(\mathrm{p}>0.05)$. On the other hand, a greater improvement was noted at 6 months in patients with KL grade 2 compared to grade 3 ; the differences within groups for most of the parameters studied reached statistical significance at 6 months but not at 12 months. We also examined if there were any differences in the responses that could be attributed to the approach used for the injection. A significant $(\mathrm{p}<0.05)$ and clinically relevant reduction compared to baseline was observed for each approach. Greater improvements were evidenced in patients administered the treatment via a medial route, although the low number of patients treated meant the differences between the three approaches were not statistically significant $(\mathrm{p}>0.05)$.

A total of 14 patients who met the protocol retreatment criteria received a second injection at the 6-month visit and were followed for another 6 months until the end of 
Table 2 Efficacy Results at 6 and 12 Months

\begin{tabular}{|c|c|c|c|c|}
\hline \multirow[t]{2}{*}{ Outcome } & \multicolumn{2}{|c|}{6 Months } & \multicolumn{2}{|c|}{12 Months } \\
\hline & mITT $(n=98)$ & $P P(n=8 I)$ & mITT (n=98) & PP $(n=74)$ \\
\hline \multicolumn{5}{|l|}{ Pain (\% change from baseline) } \\
\hline Mean (SD) & $-39.74 *(4.78)$ & $-4 I .0 I *(4.87)$ & $-37.67 *(5.11)$ & $-40.8 \mathrm{I} *(5.38)$ \\
\hline $95 \% \mathrm{Cl}$ & $(-49.23 ;-30.25)$ & $(-50.68 ;-31.34)$ & $(-47.82 ;-27.52)$ & $(-51.54 ;-30.08)$ \\
\hline \multicolumn{5}{|c|}{ WOMAC (\% Change from baseline) } \\
\hline WOMAC total mean (SD) & $-36.57^{*}(4.63)$ & $-37.53 *(4.72)$ & $-36.47 *(4.91)$ & $-40.69 *(5.09)$ \\
\hline $95 \% \mathrm{Cl}$ & $(-45.77 ;-27.37)$ & $(-46.91 ;-28.14)$ & $(-46.20 ;-26.73)$ & $(-50.84 ;-30.54)$ \\
\hline WOMAC pain mean (SD) & $-32.92 *(5.27)$ & $-33.40 *(5.44)$ & $-32.07 *(5.60)$ & $-35.97 *(6.00)$ \\
\hline $95 \% \mathrm{Cl}$ & $(-43.37 ;-22.46)$ & $(-44.22 ;-22.59)$ & $(-43.19 ;-20.95)$ & $(-47.93 ;-24.00)$ \\
\hline WOMAC stiffness mean (SD) & $-35.24^{\&}(9.05)$ & $-33.60^{\#}(9.85)$ & $-34.08 *(7.67)$ & $-34.93 *(9.31)$ \\
\hline $95 \% \mathrm{Cl}$ & $(-53.22 ;-17.23)$ & $(-53.18 ;-14.01)$ & $(-49.30 ; 18.86)$ & $(-53.49 ;-16.37)$ \\
\hline WOMAC function mean (SD) & $-33.95 *(4.81)$ & $-33.33 *(5.12)$ & $-32.7 \mid *(5.08)$ & $-39.18^{*}(4.88)$ \\
\hline $95 \% \mathrm{Cl}$ & $(-43.49 ;-24.40)$ & $(-43.49 ;-23.16)$ & $(-42.80 ;-22.62)$ & $(-48.91 ;-29.44)$ \\
\hline MCII n (\%) & $65(66.33)$ & $6 \mathrm{I}(68.54)$ & $6 \mathrm{I}(62.24)$ & $49(66.22)$ \\
\hline PGA (much better/better) n (\%) & $65(66.33)$ & $60(67.42)$ & $55(56.12)$ & $46(62.16)$ \\
\hline IGA (much better/better) n (\%) & $70(7 \mid .42)$ & $66(74.16)$ & $55(56.13)$ & 47 63.5I) \\
\hline
\end{tabular}

Note: ${ }^{*}<<0.0001 ;{ }^{{ }_{p}}=0.0002 ;{ }^{*} \mathrm{p}=0.0010$.

Abbreviations: mITT, Modified Intention to Treat; PP, per protocol; SD, standard deviation; Cl, confidence interval; WOMAC, Western Ontario and McMaster Universities Osteoarthritis Index; MCII, minimally clinical important improvement; PGA, patient global assessment; IGA, investigator global assessment.

the study. Six months after the second injection the mean reduction of pain in this group was $24.02 \%(\mathrm{p}<0.05)$ and $50 \%$ of the group achieved MCII.

Six patients discontinued the study prematurely of their own accord due to a lack of expected response, three from the mITT population and the other three only belonged to the safety population as they had not completed a post-baseline assessment. Using the median study duration until dropout as an estimator of treatment efficacy, in this group of patients the treatment was effective for just over 5 months (5.29 months).

At the 12-month visit, almost $47 \%$ of the patients reported having taken paracetamol as rescue medication at some point during the follow-up period.

\section{Safety Results}

A total of 12 treatment-related adverse events were reported involving eight patients $(7.9 \%)$. These adverse events were all local, of mild-to-moderate intensity and classified as non-serious. No treatment-related adverse events were reported after the second injection. No serious treatment-related adverse events were reported throughout the overall study period.

\section{Discussion}

Viscosupplementation is a recognised treatment for $\mathrm{OA}$ in different joints. Several systematic reviews and metaanalyses advocate its use in terms of efficacy and safety $^{11,13,16-19,21,24,27,29}$ and, unlike other treatments such as oral drugs, HA is free from drug-product interactions and problems associated with compliance or the risks of overuse. ${ }^{37}$ The most widespread use of HA is in knee $\mathrm{OA}$, and although they were initially administered in cycles of 3-5 injections, HA products based on a single injection have been in demand for some time. Since the effects of HA were believed to last for 6 months, ${ }^{38,39}$ very few studies have been conducted with single-injection products and follow-up periods lasting more than 6 months. Considering this background, after confirming the biocompatibility of MPS-HA $2 \%,{ }^{40}$ our objective was to study its efficacy and safety in patients with symptomatic knee OA for 1 year after a single injection.

The results suggest that MPS-HA2\% is effective in relieving pain and improving function. The reduction of VAS pain at 6 months was significant compared to baseline and lasted for the whole follow-up period with relative reductions ranging from $37.67 \%$ to $41.01 \%$. Significant 
improvements were also observed in WOMAC scores (A, $\mathrm{B}, \mathrm{C}$ and total) with improvements ranging from $32.07 \%$ to $40.69 \%$ for the 12 -month follow-up period; these results are well above the $20 \%$ considered as clinically relevant. ${ }^{16}$ After 6 months, $66.33 \%$ of patients achieved MCII and by 12 months this value was $62.24 \%$. The patient and investigator global assessments followed a similar pattern.

Patient retention is a point of concern in clinical trials, especially long-term studies. ${ }^{41}$ In our study, the required sample size was estimated considering an expected dropout rate of $35 \%$ throughout the entire study period; therefore, a discontinuation rate of $18 \%$ after 12 months' follow-up can be considered a good result that was probably due to the efficacy and tolerability of the treatment.

One of the keys to viscosupplementation is the correct identification of the patient characteristics that may be associated with a better response. Obesity and a high radiographic grade have been identified as predictors of viscosupplementation failure. ${ }^{42-44}$ Consequently, HA administration to obese patients and those with more severe grades of knee OA has frequently been discouraged. However, more recent studies have concluded that while these patients may have a reduced possibility of a positive response to $\mathrm{HA}$ treatment, the benefits obtained in those who do respond are similar to those of normal weight subjects and with mild/moderate OA. ${ }^{45}$ Although our study did not include obese patients, about $80 \%$ of our sample were overweight and we did not find significant differences in the response rates between overweight and normal or underweight patients. On the other hand, when considering the grade of OA, we found differences in some of the response parameters at 6 months but not after 12 months. As such, given there are very few effective and well-tolerated treatment options for OA, we share the opinion of most recent publications in that HA should not be ruled out as a therapeutic option for these patients because a substantial number can benefit from it.

Intra-articular injections can be administered via several approaches, and different outcomes have been reported in terms of efficacy and local reactions. ${ }^{46-48}$ In the present study the protocol did not specify the approach to be used and each investigator administered the treatment according to their own judgement and experience. The superolateral and lateral approaches were used in a similar number of patients, whereas the medial route was much less frequent. Although greater improvements were observed for patients treated via the medial approach, the reduced number of subjects meant the differences between groups were not statistically significant and the results should be considered with caution.

Just $14 \%$ of the patients received a second, single injection at 6 months and were followed for another 6 months, going on to record a clinically important improvement in pain and function and confirm that clinical outcomes can be improved by repeating HA administration if the symptoms reappear. ${ }^{19,49-52}$

With respect to safety, a single injection of MPS-HA2 $\%$ was well tolerated and only 8 patients reported local, treatment-related adverse events. These adverse events were consistent with those commonly described for this class of products and none were serious.

The limitations of this study include the open-label design without a direct comparator arm. With regard to the possibility of a head-to-head design, we could not find any controlled trials with single-injection HA products that would have demonstrated 12-month efficacy in a significant number of patients.

Another limitation is due to the fact that although all our patients had predominantly tibiofemoral compromise, a significative percentage presented some degree of patellofemoral affectation. This could be a source of bias as patellofemoral involvement could lead to worse efficacy results, ${ }^{14,53}$ so many studies exclude these patients. The inclusion of purely tibiofemoral patients may have resulted in even better results, although we consider that our patient sample is a better reflection of the real situation.

\section{Conclusions}

The results of this study suggest that a single injection of MPS-HA2\% provides long lasting improvement in patients with mild to moderate knee OA but further controlled trials must be performed to confirm or infirm these promising data. Patient tolerability and safety were both optimal.

\section{Data Sharing Statement}

The authors have no objection in sharing the datasets related to this study. Requests should be made to the corresponding author.

\section{Acknowledgments}

We would like to thank Isabel Camacho from Alpha Bioresearch for the statistical analysis of the results. 


\section{Author Contributions}

All authors made significant contributions to the work reported, whether that is in the conception, study design, execution, acquisition of data, analysis and interpretation, or in all of these areas; took part in drafting, revising or critically reviewing the article; gave final approval of the version to be published; have agreed on the journal to which the article has been submitted; and agree to be accountable for all aspects of the work.

\section{Disclosure}

F. J. Blanco report grants, personal fees from Meiji Pharma Spain. P. Coronel and M. Gimeno are employees of Meiji Pharma Spain. The other authors report no conflict of interest in this work.

\section{References}

1. Herrero-Beaumont G, Pérez-Baos S, Sánchez-Pernaute O, RomanBlas JA, Lamuedra A, Largo R. Targeting chronic innate inflammatory pathways, the main road to prevention of osteoarthritis progression. Biochem Pharmacol. 2019;165:24-32.

2. Chen D, Shen J, Zhao W, et al. Osteoarthritis: toward a comprehensive understanding of pathological mechanism. Bone Res. 2017;17(5):16044.

3. Cooper C, Rannou F, Richette P, et al. Use of intraarticular hyaluronic acid in the management of knee osteoarthritis in clinical practice. Arthritis Care Res (Hoboken). 2017;69(9):1287-1296.

4. Deveza LA, Melo L, Yamato TP, Mills K, Ravi V, Hunter DJ. Knee osteoarthritis phenotypes and their relevance for outcomes: a systematic review. Osteoarthritis Cartilage. 2017;25:1926-1941.

5. Dell'Isola A, Allan R, Smith SL, Marreiros SS, Steultjens M. Identification of clinical phenotypes in knee osteoarthritis: a systematic review of the literature. BMC Musculoskelet Disord. 2016;17:42.

6. Hunter DJ, Schofield D, Callander E. The individual and socioeconomic impact of osteoarthritis. Nat Rev Rheumatol. 2014;10 (7):437-441

7. Hunter DJ, Bierma-Zeinstra S. Osteoarthritis. Lancet. 2019;393 (10182):1745-1759.

8. Migliore A, Gigliucci G, Alekseeva L, et al. Treat-to-target strategy for knee osteoarthritis. International technical expert panel consensus and good clinical practice statements. Ther Adv Musculoskelet Dis 2019;19(11):1759720X19893800.

9. Bowman S, Awad ME, Hamrick MW, Hunter M, Fulzele S. Recent advances in hyaluronic acid-based therapy for osteoarthritis. Clin Transl Med. 2018;7(1):6.

10. Balazs EA, Denlinger JL. Viscosupplementation: a new concept in the treatment of osteoarthritis. J Rheumatol Suppl. 1993;39:3-9.

11. Altman RD, Manjoo A, Fierlinger A, Niazi F, Nicholls M. The mechanism of action for hyaluronic acid treatment in the osteoarthritic knee: a systematic review. BMC Musculoskelet Disord. 2015;26(16):321.

12. Trojian TH, Concoff AL, Joy SM, Hatzenbuehler JR, Saulsberry WJ, Coleman CI. AMSSM scientific statement concerning viscosupplementation injections for knee osteoarthritis: importance for individual patient outcomes. Br J Sports Med. 2016;50 (2):84-92.
13. Bannuru RR, Schmid CH, Kent DM, Vaysbrot EE, Wong JB, McAlindon TE. Comparative effectiveness of pharmacologic interventions for knee osteoarthritis: a systematic review and network meta-analysis. Ann Intern Med. 2015;162(1):46-54.

14. Conrozier T, Monfort J, Chevalier X, et al. EUROVISCO recommendations for optimizing the clinical results of viscosupplementation in osteoarthritis. Cartilage. 2018;1:1947603518783455.

15. Bannuru RR, Osani MC, Vaysbrot EE, et al. OARSI guidelines for the non-surgical management of knee, hip, and polyarticular osteoarthritis. Osteoarthritis Cartilage. 2019;27(11):1578-1589.

16. Concoff A, Rosen J, Fu F, et al. A comparison of treatment effects for nonsurgical therapies and the minimum clinically important difference in knee osteoarthritis: a systematic review. JBJS Rev. 2019;7(8):e5.

17. Richette P, Chevalier X, Ea HK, et al. Hyaluronan for knee osteoarthritis: an updated meta-analysis of trials with low risk of bias. $R M D$ Open. 2015;1(1):e000071.

18. Strand V, McIntyre LF, Beach WR, Miller LE, Block JE. Safety and efficacy of US-approved viscosupplements for knee osteoarthritis: a systematic review and meta-analysis of randomized, saline-controlled trials. J Pain Res. 2015;8:217-228.

19. Maheu E, Rannou F, Reginster JY. Efficacy and safety of hyaluronic acid in the management of osteoarthritis: evidence from real-life setting trials and surveys. Semin Arthritis Rheum. 2016;45(4 Suppl): S28-33.

20. Henrotin Y, Raman R, Richette P, et al. Consensus statement on viscosupplementation with hyaluronic acid for the management of osteoarthritis. Semin Arthritis Rheum. 2015;45(2):140-149.

21. Maheu E, Bannuru RR, Herrero-Beaumont G, Allali F, Bard H, Migliore A. Why we should definitely include intra-articular hyaluronic acid as a therapeutic option in the management of knee osteoarthritis: results of an extensive critical literature review. Semin Arthritis Rheum. 2019;48(4):563-572.

22. Bannuru RR, Natov NS, Obadan IE, Price LL, Schmid CH, McAlindon TE. Therapeutic trajectory of hyaluronic acid versus corticosteroids in the treatment of knee osteoarthritis: a systematic review and meta-analysis. Arthritis Rheum. 2009;61(12):1704-1711.

23. Yaftali NA, Weber K. Corticosteroids and hyaluronic acid injections. Clin Sports Med. 2019;38(1):1-15.

24. He WW, Kuang MJ, Zhao J, et al. Efficacy and safety of intraarticular hyaluronic acid and corticosteroid for knee osteoarthritis: a meta-analysis. Int J Surg. 2017;39:95-103.

25. Bisicchia S, Tudisco C. Hyaluronic acid vs corticosteroids in symptomatic knee osteoarthritis: a mini-review of the literature. Clin Cases Miner Bone Metab. 2017;14(2):182-185.

26. Wang F, He X. Intra-articular hyaluronic acid and corticosteroids in the treatment of knee osteoarthritis: a meta-analysis. Exp Ther Med. 2015;9(2):493-500. doi:10.3892/etm.2014.2131

27. Charlesworth J, Fitzpatrick J, Perera NKP, Orchard J. Osteoarthritisa systematic review of long-term safety implications for osteoarthritis of the knee. BMC Musculoskelet Disord. 2019;20(1):151.

28. Conaghan PG, Arden N, Avouac B, Migliore A, Rizzoli R. Safety of paracetamol in osteoarthritis: what does the literature say? Drugs Aging. 2019;36(Supp1 1):7-14.

29. Bannuru RR, Osani M, Vaysbrot EE, McAlindon TE. Comparative safety profile of hyaluronic acid products for knee osteoarthritis: a systematic review and network meta-analysis. Osteoarthritis Cartilage. 2016;24(12):2022-2041.

30. Kompel AJ, Roemer FW, Murakami AM, Diaz LE, Crema MD, Guermazi A. Intra-articular corticosteroid injections in the hip and knee: perhaps not as safe as we thought? Radiology. 2019;293 (3):656-663.

31. Maheu E, Avouac B, Dreiser RL, Bardin T. A single intra-articular injection of $2.0 \%$ non-chemically modified sodium hyaluronate vs $0.8 \%$ hylan G-F 20 in the treatment of symptomatic knee osteoarthritis: a 6-month, multicenter, randomized, controlled non-inferiority trial. PLoS One. 2019;14(12):e0226007. 
32. Vincent P. Intra-articular hyaluronic acid in the symptomatic treatment of knee osteoarthritis: a meta-analysis of single-injection products. Curr Ther Res Clin Exp. 2019;7(90):39-51.

33. Berenbaum F, Grifka J, Cazzaniga S, et al. A randomised, double-blind, controlled trial comparing two intra-articular hyaluronic acid preparations differing by their molecular weight in symptomatic knee osteoarthritis. Ann Rheum Dis. 2012;71 (9):1454-1460.

34. Altman R, Asch E, Bloch D, et al. Development of criteria for the classification and reporting of osteoarthritis. Classification of osteoarthritis of the knee. Diagnostic and therapeutic criteria Committee of the American Rheumatism Association. Arthritis Rheum. 1986;29 (8):1039-1049.

35. Kellgren JH, Lawrence JS. Radiological assessment of osteo-arthrosis. Ann Rheum Dis. 1957;16(4):494-502.

36. Tubach F, Ravaud P, Martin-Mola E, et al. Minimum clinically important improvement and patient acceptable symptom state in pain and function in rheumatoid arthritis, ankylosing spondylitis, chronic back pain, hand osteoarthritis, and hip and knee osteoarthritis: results from a prospective multinational study. Arthritis Care Res (Hoboken). 2012;64(11):1699-1707.

37. Vangsness CT Jr, Adamson TC 3rd, Daley MJ. Consequences on private insurance coverage: the AAOS clinical practice guidelines and hyaluronic acid injections. J Bone Joint Surg Am. 2020;102 (10):920-926.

38. Bruyère $\mathrm{O}$, Cooper $\mathrm{C}$, Pelletier JP, et al. A consensus statement on the European Society for Clinical and Economic Aspects of Osteoporosis and Osteoarthritis (ESCEO) algorithm for the management of knee osteoarthritis-From evidence-based medicine to the real-life setting. Semin Arthritis Rheum. 2016;45(4 Suppl):S3-11.

39. Raman R, Henrotin Y, Chevalier X, et al. Decision algorithms for the retreatment with viscosupplementation in patients suffering from knee osteoarthritis: recommendations from the EUROpean VIScosupplementation Consensus Group (EUROVISCO). Cartilage. 2018;9(3):263-275.

40. Largo R, López-Reyes AG, Medina JP, Gimeno M, Coronel P, Herrero-Beaumont G. Concentrated hyaluronic acid intraarticular injection is well tolerated by osteoarthritic joint and prevents pro-inflammatory mechanisms in the osteoarthritic synovium. World Congress on Osteoporosis, Osteoarthritis and Musculoskeletal Diseases (WCO-IOF-ESCEO 2019): posters Abstracts. Osteoporos Int. 2019;30:253-773.

41. Mazzuca SA, Brandt KD, Katz BP, et al. Subject retention and adherence in a randomized placebo-controlled trial of a disease-modifying osteoarthritis drug. Arthritis Rheum. 2004;51 (6):933-940.
42. Conrozier T, Monfort J, Chevalier X, et al. EUROVISCO recommendations for optimizing the clinical results of viscosupplementation in osteoarthritis. Cartilage. 2020;11(1):47-59.

43. Eymard F, Chevalier X, Conrozier T. Obesity and radiological severity are associated with viscosupplementation failure in patients with knee osteoarthritis. J Orthop Res. 2017;35(10):2269-2274.

44. Altman RD, Farrokhyar F, Fierlinger A, Niazi F, Rosen J. Analysis for prognostic factors from a database for the intra-articular hyaluronic acid (Euflexxa) treatment for osteoarthritis of the knee. Cartilage. 2016;Jul(3):229-237.

45. Conrozier T, Eymard F, Chouk M, Chevalier X. Impact of obesity, structural severity and their combination on the efficacy of viscosupplementation in patients with knee osteoarthritis. BMC Musculoskelet Disord. 2019;20(1):376.

46. D'Alessandro R, Falsetti P, Conticini E, et al. Difference in pain and accuracy of two hyaluronic acid injection techniques for symptomatic knee osteoarthritis in overweight patients. Reumatologia. 2021;59 (1):23-26.

47. Hermans J, Bierma-Zeinstra SM, Bos PK, Verhaar JA, Reijman M. The most accurate approach for intra-articular needle placement in the knee joint: a systematic review. Semin Arthritis Rheum. 2011;41(2):106-115.

48. Lee SY, Gn KK, Chung BJ, Lee SW, Kim TK. Anterolateral portal is less painful than superolateral portal in knee intra-articular injection. Knee Surg Relat Res. 2015;27(4):228-232.

49. Altman R, Hackel J, Niazi F, Shaw P, Nicholls M. Efficacy and safety of repeated courses of hyaluronic acid injections for knee osteoarthritis: a systematic review. Semin Arthritis Rheum. 2018;48(2):168-175.

50. Navarro-Sarabia F, Coronel P, Collantes E, Navarro FJ, de la Serna AR, Naranjo A. A 40-month multicentre, randomised placebo-controlled study to assess the efficacy and carry-over effect of repeated intra-articular injections of hyaluronic acid in knee osteoarthritis: the AMELIA project. Ann Rheum Dis. 2011;70:1957-1962.

51. Pal S, Thuppal S, Reddy KJ, et al. Long-term (1-Year) safety and efficacy of a single 6-mL injection of Hylan G-F 20 in Indian patients with symptomatic knee osteoarthritis. Open Rheumatol J. 2014;8:54-68.

52. Altman RD, Rosen JE, Bloch DA, Hatoum HT. Safety and efficacy of retreatment with a bioengineered hyaluronate for painful osteoarthritis of the knee: results of the open-label Extension Study of the FLEXX Trial. Osteoarthritis Cartilage. 2011;19(10):1169-1175.

53. Conrozier T, Mathieu P, Schott AM, et al. Factors predicting long-term efficacy of Hylan GF-20 viscosupplementation in knee osteoarthritis. Joint Bone Spine. 2003;70(2):128-133.
Journal of Pain Research

\section{Publish your work in this journal}

The Journal of Pain Research is an international, peer reviewed, open access, online journal that welcomes laboratory and clinical findings in the fields of pain research and the prevention and management of pain. Original research, reviews, symposium reports, hypothesis formation and commentaries are all considered for publication. The manuscript

Submit your manuscript here: https://www.dovepress.com/journal-of-pain-research-journal management system is completely online and includes a very quick and fair peer-review system, which is all easy to use. Visit http:// www.dovepress.com/testimonials.php to read real quotes from published authors. 